\title{
A Study On Peritoneal Fluid Culture And Its Antibiotic Sensitivity In Perforative Peritonitis Cases
}

\author{
Dr.J.Ravisankar ${ }^{1}$, Dr. V.S Venkatesan ${ }^{1}$ \\ (Department Of General Surgery,Madurai Medical College And Hospital, India) \\ (Department Of General Surgery, Coimbatore Medical College And Hospital, India)
}

\begin{abstract}
:
Aim: To analyse bacteriological and its sensitivity pattern in peritoneal fluid in cases of perforative peritonitis admitting in $\mathrm{CMCH}$ and $\mathrm{MMCH}$ so as to select appropriate empirical antibiotic therapy.

Methods: Patients presenting to the casualty of $\mathrm{CMCH}$ and $\mathrm{MMCH}$ with features of Perforative peritonitis such as abdominal guarding, rigidity and Chest Radiograph showing bilateral air under diaphragm are taken up for emergency laparotomy and the peritoneal fluid is sent for bacterial culture and sensitivity and according to the laboratory results appropriate antibiotics are initiated in the post-operative period.

Results: From this study it was analysed that the most common organism isolated from peritoneal fluid of perforative peritonitis patients is Klebsiella species and second most common was Escherichia Coli. Appropriate antibiotics such as cephalosporins were started for the patient according to the culture and sensitivity reports.

Conclusion: In this study, it is concluded that perforation most commonly seen in duodenum followed by stomach. Most of the cases were due to peptic ulcer disease. Secondary peritonitis caused in these cases was most commonly due to Klebsiella followed by Escherichia coli and rarely by mixed and proteus and pseudomonas. Both Klebsiella and Escherichia coli were sensitive to cephalosporin group of drugs followed by quinolones followed by macrolide antibiotics.
\end{abstract}

Keywords: Perforative peritonitis, Klebsiella, Escherichia coli, Cephalosporins

\section{Introduction}

Surgical peritonitis remains one of the most common problems faced by a surgeon. Whether it is a simple duodenal perforation, traumatic perforation, appendicular perforation, or a case of acute pancreatitis complicated by a pancreatic abscess, it still remains as a major cause of morbidity and mortality. Only in recent decades there has been significant improvement in treatment of peritonitis both by use of antibiotic and surgery.

Intra abdominal infection is a major challenge to the surgeon. Peritonitis which we commonly come to us is secondary peritonitis occurring due to perforation of hollow viscus. The surgeons treating it know the dreadful and fatal complication, the problems can be minor wound infection to dangerous septic shock or SIRS (systemic inflammatory response syndrome).

\section{There are various obstacles for treatment of peritonitis they include}

- The age of patient

- Time interval of presentation

- General condition and nutritional status of patient

- Presence of any malignancy

- Post operative complications

The other fact which makes peritonitis more dangerous is due the very high amount of contamination of the peritoneal cavity by certain fatal organisms belonging to the enterobacteriaceae family. These include E.coli , klebsiellla, proteus, enterococci species. These organisms directly or by their toxins cause certain effects which leads to the onset of SIRS. Alteimer in the $1930 \mathrm{~s}$ isolated polymicrobial organisms from the peritoneal fluid and also showed that their pathogenicity is causing intra abdominal sepsis

The antibiotic treatment of intra abdominal infection has evolved for the period of past 30 years and is based on solid experimental and class 1 clinical data. In fact the earliest experiment done by Weinstein et al showed that a combined therapy targeted towards both aerobic and anaerobic organisms was regarded as correct regarding survival and helped minimizing abscess formation.Current therapy towards the treatment of peritonitis is directed at correction of underlying cause, administration of systemic antibiotics, and facilitating supportive therapy to prevent formation of SIRS.

With antibiotic administration it was found that if the therapy was directed towards aerobes there was lesser mortality and more residual abscess formation but when therapy was directed towards anaerobes there 
was less abscess formation and mortality remained unchanged. Therefore therapy was considered optimal when combination was used.

The treatment can be done easily by starting a certain line of antibiotic therapy these usually include a broad spectrum antibiotic that covers gram positive, gram negative and anaerobes. However the problem now is the development of resistance to these antibiotics and results in high failure rates in the treatment .

In this study, various organisms that are growing in the peritoneal fluid culture of the patients presenting with perforative peritonitis and their antibiotic sensitivity and resistance pattern were analysed, So that we can initiate early and appropriate antibiotic therapy to patients presenting with perforative peritonitis preoperatively which can improve the outcome of the patient.

\section{Background and purpose of the study:}

To analyse bacteriological and its sensitivity pattern in peritoneal fluid in cases of perforative peritonitis admitting in $\mathrm{CMCH}$, so as to select appropriate empirical antibiotic therapy.

1. To study bacteriological pattern in peritoneal fluid by culture.

2. To determine antibiotic sensitivity and resistance pattern for commonly used antibiotics to the organisms grown in culture.

\section{Problem statement:}

Perforation peritonitis is one of the commonest surgical emergency we come across in our practice. Though there are many recent advances in the field of medicine, perforation peritonitis still has a major threat to the surgeon. The major problem faced by the surgeons is the late presentation of patient to the doctor and development of resistance bacterial organisms that causes peritonitis and sepsis. With increasing risk of emerging drug resistance to antibiotics, this problem has to be taken care of in rapid manner.

\section{Definition:}

Peritonitis is inflammation of the peritoneal covering the abdominal cavity. The aetiology of peritonitis may vary but the outcome of it remains same in all cases. Peritonitis is also used interchangeably with intrabdominal sepsis. The most common peritonitis we come across in surgical practice is perforation peritonitis either infective or traumatic etiology or postoperative following anastomotic leak.Peritonitis is of three types, 1. Primary 2. Secondary 3. Tertiary.Primary peritonitis is defined as bacterial infection of peritoneal cavity that occurs arising from extraperitoneal site possibly lymphatic or hematogenous spread. It occurs most commonly in alcoholic cirrhosis with ascites and in nephritic syndrome. Presence of ascites increases the chance of developing peritonitis due to low protein concentration.Secondary peritonitis occurs as a result of contamination from intraperitoneal organ within peritoneal cavity. Most of the cases occurs as result of primary lesion in duodenum, stomach and appendix. $10 \%$ cases of secondary peritonitis occurs as a complication of abdominal surgery.Tertiary peritonitis refers to persistent diffuse peritonitis that occurs after initial treatment for secondary peritonitis. It appears to represent both failure of host response and superinfection.

\section{Peritoneal fliud culture:}

For isolation of organisms and identification of resistant organisms from peritoneal fluid culture were practised since 1930, where altemeier cultures the infective fluid in perforated appendix and showed heavy contamination of aerobic and anaerobic infection on culture. Though anerobic and aerobic organisms were identified in peritoneal fluid, these findings were found to represent either single or only couple of organisms. But with the recent observations both aerobic and anaerobic organisms can work in synergy to promote the pathogenicity of the infective process.

Moreover the practice of obtaining intraoperative peritoneal fluid for culture in patients with abdominal infections has been considered as important in providing the basis for choices and changes in postoperative antibiotic therapy. However recent studies challenged the practice and role of peritoneal fluid culture is controversial. Till today practice antibiotic resistance is occurring at a significant rate and often associated with clinical failure. Infection of peritoneal cavity following perforation results in contamination of peritoneal cavity with bacteria, which is treated with conventional antibiotics therapy, is complicated by both by the emergence of antibiotic resistance and increased patient population intrinsically at risk for nosocomial infections. All above factors makes the surgeon to do peritoneal fliud culture intraoperatively. Avery demonstrated that there is an increasing evidence for drug resistance to empirical drug therapy for organisms isolated in peritoneal fluid. The need for reducing antibiotic exposure and value of knowledge obtained from observing microbial sensitivity pattern support routine need for peritoneal fluid culture.

A study conducted by Martin. J. Bell et al demonstrated polybacterial contamination of peritoneal cavity in more than half of neonates in whom cultures were taken. $23 \%$ of culture showed to have a mixed aerobic and anerobic flora. He showed the common organism isolated were E.coli and bacteroides species. Another study 
done by John Boey showed E.coli, Klebsiella and pseudomonas were most common isolates in perforated duodenal ulcer where perforation has occurred two days before. In other two studies E.coli is demonstrated as most common organism in peritoneal fluid culture in perforation peritonitis. In study conducted by Mutiibwa, demonstrated that in order to guide in choice of antimicrobial therapy, peritoneal fluid culture in perforation peritonitis is necessary.

\section{Materials And Methods}

Design Of Study: Cross sectional study

Place Of Study: Coimbatore Medical College and Hospital and Madurai Medical college and hospital.

Study Period: July 2014-June 2015

Study Population: Patients presenting to Coimbatore and Madurai Medical college hospital with perforation peritonitis.

sample size: 50

Inclusion criteria:

1. Patient presenting with features of perforation peritonitis and confirmed by $\mathrm{x}$ ray

2. Age more than 18 yrs

Exclusion criteria:

1. Patient presenting with primary peritonitis

2. Peritonitis due to trauma

\section{Pre Operative Evaluation}

\section{Methodology}

Patients with features of perforation peritonitis presenting to casualty to Coimbatore medical college were admitted. Following which detailed history were taken and complete physical examination were done and diagnosis is confirmed using chest and abdomen $\mathrm{X}$ ray erect which shows air under diaphragm. Following which routine investigations like $\mathrm{CBC}$, Blood urea and sugar and serum creatinine and electrolytes and ECG were done. Patients were excluded on the basis of above criteria. Following confirmation of diagnosis patients were planned for emergency laparotomy and perforation closure.

\section{Preoperative Preparation}

Patient confirmed with diagnosis of perforation peritonitis were resuscitated with intravenous fluid and stabilising the patient vitals were planned for emergency laparotomy and taken up for surgery after getting consent from the patient and his/ her attenders.

\section{Intraoperative Procedure}

Emergency laparotomy done using midline incision and peritoneal fluid was obtained from confirmed non traumatic cases and sent for aerobic microbiological culture. Following which perforation closure is done using vicryl with live omental patch and abdomen is closed after keeping abdominal drains.

\section{Post Operative Care}

Following surgery patient were given routine postoperative care with intravenous fluids and antibiotics. Peritoneal fluid culture reports were followed up and the isolated organisms were tested for antimicrobial sensitivity by Kirby-Bauer disc diffusion method using ampicillin, amikacin, ciprofloxacin, ceftriaxone and cotrimoxazole and the culture reports were obtained. Antibiotics were changed according to the sensitivity pattern of organism grown in the culture.

\section{Discussion}

Secondary peritonitis caused by hollow viscus perforation is common. It has high mortality rate due to late presentation of patient to hospital. In our study secondary peritonitis due to perforation was common in males than females, which is in the ratio of 7:1. And this ratio is slightly higher in our study when compared to other standard literature. Most cases of perforation seen in case of males which is probably due to their irregular food habits, alcoholism and smoking. In our study most of the cases of perforation were seen in the age group of $31-40 y r s$ followed by $20-30$ yrs. The mean age of presentation is $35.26 \mathrm{yrs}$ of age. Most of the patients have previous history of peptic ulcer disease. There in is no exposure of drugs like steroids and NSAID's in long term as confirmed by history from the patient. Regarding the presentation of patient to hospital, majority of patient reaches the hospital 2-3 days of symptoms which roughly $50 \%$ of cases. Only $11 \%$ of patients with perforation peritonitis present to us within 1 day of symptom. The mean duration of presentation is about 2.6 days. The 
delay in presentation of patient to may be due to ignorance and lack of convenience to the hospital care

From our study, it has been noticed that the most common site of perforation is in $2^{\text {nd }}$ part of duodenum $52 \%$ followed by gastric in $42 \%$ of cases. Most are likely of peptic ulcer in origin. Only about 3 cases i.e $6 \%$ cases were due to ileal perforation and are of non typhoid in origin.In this study, peritoneal fluid culture sent for aerobic microbial culture shows monomicrobial growth in $84 \%$ cases, polymicrobial in $2 \%$ cases and no growth in $14 \%$ cases. Gram negative enteric bacilli were being common in the culture and this includes E coli and Klebsiella followed by proteus and pseudomonas. The most common organism grown were Klebsiella $46 \%$ followed by E coli in $34 \%$ of cases only $2 \%$ showed mixed growth of both E coli and Klebsiella. In about 7 cases i.e $14 \%$ showed no growth in their culture. Most of the culture negative cases, presented to us within one day of symptoms of perforation and this shows that initial peritonitis was due to chemical peritonitis. In our study, the sensitivity patterns of cultured organisms were analysed. It showed that organisms were sensitive in most cases to ceftriaxone followed by ciprofloxacin and amikacin. But these organisms showed high resistance to ampicillin and cotrimoxazole. E coli cultured in peritonitis in our study showed sensitivity to ceftriaxone of about $87.5 \%$ followed by ciprofloxacin and amikacin of about $81.25 \%$.

In case of Klebsiella, the sensitivity to ceftriaxone is $91.07 \%$, followed by amikacin which is about $78 \%$ and ciprofloxacin $73.9 \%$. Both $\mathrm{E}$ coli and Klebsiella showed high resistance to ampicilin and cotrimoxazole. We have noticed mild increase in resistance to ciprofloxacin and amikacin group of drugs.Metronidazole in the treatment of anaerobic bacterial infection still holds good. Development of resistance to metronidazole among anaerobes is still very low and is confirmed in many studies. But development of resistance to aerobic bacteria is on the rise, due to inadvertent use of antibiotics. Due to this fact there has been confusion in selecting the empirical antibiotic therapy.From this study, it concludes that drug that is most sensitive in most of cases of perforation peritonitis is cephalosporins followed by quinolones and amikacin group of drugs. Most of the cases showed resistance to ampicillin and cotrimoxazole group drugs.

\section{Conclusion}

In this study, it is concluded that perforation most commonly seen in duodenum followed by stomach. Most of the cases were due to peptic ulcer disease. Secondary peritonitis caused in these cases was most commonly due to Klebsiella followed by Escherichia coli and rarely by mixed and proteus and pseudomonas. Both Klebsiella and Escherichia coli were sensitive to cephalosporin group of drugs followed by quinolones followed by macrolide antibiotics.

\section{References}

[1]. MAINGOT'S abdominal surgery, $10^{\text {th }}$ edition, pg no: $633-650$

[2]. SABISTON textbook of surgery, $19^{\text {th }}$ edition, pg n: $1088-1114$

[3]. SCHWARTZ'S principle of surgery, $10^{\text {th }}$ edition, pg no: 1035-1099

[4]. T.S. RANGANATHAN textbook of anatomy, $6^{\text {th }}$ edition, pg no: 273-281

[5]. BAVEJA, textbook of microbiology, $3^{\text {rd }}$ edition, pg no: $312-340$

[6]. TRIPATHI, textbook of pharmacology, $5^{\text {th }}$ edition, pg no: 627-697

[7]. SRB, manual of surgery, $4^{\text {th }}$ edition, pg no: 599-614

[8]. Aerobic bacterial causes of secondary peritonitis and their sensitivity pattern in non traumatic small bowel perforation, D. Mutiibwa et al, East cent Afr J Surg, july 2013

[9]. Whether culture positivity and perforation operation interval affects mortality in perforation peritonitis?, Dr. aslam, et al, Indian Journal of Basic and applied medical research, March 2015, volume 4

[10]. Appendectomy in paediatrics the value of peritoneal fluid smear and its bacteriological profile, Dr. Manal, et 1, Open journal of medical microbiology, 2012, 2

[11]. Peritoneal fluid culture and antibiotic treatment in patients with perforated appendicitis in pacific island, Dr. Alexia et al, Asian Journal of surgery $2015,1-5$

[12]. Non traumatic terminal ileal perforation, Dr. Rauf, et al, World journal of emergency surgery

[13]. Metronidazole is still the drug of choice for treatment of anerobic infection, Dr. Sonja, et al, Sweden 